Canad. Math. Bull. Vol. 19 (3), 1976

\title{
REPRESENTING HOMOLOGY CLASSES ON SURFACES
}

\author{
BY
}

JAMES A. SCHAFER

Let $T^{2}=S^{1} \times S^{1}$, where $S^{1}$ is the unit circle, and let $\{\alpha, \beta\}$ be the integral basis of $H_{1}\left(T^{2}\right)$ induced by the $2 S^{1}$-factors. It is well known that $0 \neq X=$ $p \alpha+q \beta$ is represented by a simple closed curve (i.e. the homotopy class $\alpha^{p} \beta^{q}$ contains a simple closed curve) if and only if $\operatorname{gcd}(p, q)=1$. It is the purpose of this note to extend this theorem to oriented surfaces of genus $g$.

Let $S_{\mathrm{g}}$ be an oriented surface of genus $g>0$. The fundamental group $\pi_{\mathrm{g}}$ can be presented as $\left\{a_{1}, \ldots, a_{\mathrm{g}}, b_{1}, \ldots, b_{\mathrm{g}}: \prod_{1}^{g}\left[a_{i}, b_{i}\right]\right\}$ and $H_{1}\left(S_{\mathrm{g}}\right)$ is a free abelian group with $2 \mathrm{~g}$ canonical generators, $\alpha_{1}, \ldots, \alpha_{g}, \beta_{1}, \ldots, \beta_{g}$, the images of $a_{i}, b_{i}$ respectively. Recall that a homology class $X \in H_{1}\left(S_{g}\right)$ is said to be represented by a simple closed curve if and only if there exists a homotopy class $\alpha \in \pi_{1}\left(S_{g}\right)$ containing a simple closed curve and $h(\alpha)=X$ where $h: \pi_{1}\left(S_{\mathrm{g}}\right) \rightarrow \pi_{1}\left(S_{\mathrm{g}}\right)_{a b}=$ $H_{1}\left(S_{g}\right)$ is the Hurewicz map. It is the purpose of this note to prove the following theorem.

THEOREM. Let $S_{\mathrm{g}}$ be an oriented surface of genus $g>0$, and let $\left\{\alpha_{1}, \ldots, \alpha_{g} ; \beta_{1}, \ldots, \beta_{g}\right\}$ be the canonical basis of $H_{1}\left(S_{g}\right)$. Then $0 \neq X=$ $\sum_{1}^{q} p_{i} \alpha_{i}+q_{i} \beta_{i} \in H_{1}\left(S_{\mathrm{g}}\right)$ is represented by a simple closed curve if and only if $\operatorname{gcd}\left\{p_{1}, \ldots, p_{\mathrm{g}}, q_{1}, \ldots, q_{\mathrm{g}}\right\}=1$.

Proof. Suppose $\operatorname{gcd}\left\{p_{1}, \ldots, q_{\mathrm{g}}\right\}=1$, then $X$ is part of an integral basis for $H_{1}\left(S_{\mathrm{g}}\right)$. Now $H_{1}\left(S_{\mathrm{g}}\right)$ supports a non-degenerate skew-symmetric bilinear form (the intersection pairing) and $\alpha_{1}, \ldots, \alpha_{\mathrm{g}}, \beta_{1}, \ldots, \beta_{\mathrm{g}}$ is a symplectic basis for this pairing. Since the form is skew-symmetric, every vector is isotropic, i.e. $X \cdot X=0$. From the non-degeneracy of the form, choose $Y \neq 0$ so that $X \cdot Y=$ 1. It is easy to see that the form restricted to the subspace generated by $X$ and $Y$ is non degenerate. It follows easily that $H_{1}\left(S_{g}\right) \simeq\{X, Y\} \oplus\{X, Y\}^{\perp}$. Hence $X$ is part of a symplectic basis for $H_{1}\left(S_{\mathrm{g}}\right)$. We may assume $X=M\left(\alpha_{1}\right)$ where $M$ is a symplectic transformation on $H_{1}\left(S_{g}\right)$. Now every symplectic automorphism of $H_{1}\left(S_{g}\right)$ is induced by an automorphism of $\pi_{1}\left(S_{g}\right)$. This is most easily demonstrated by exhibiting a set of generators for the symplectic automorphisms of $Z^{28}$ and showing each is induced by an automorphism of $\pi_{1}\left(S_{g}\right)$ [1]. By Nielsen's theorem [2], every automorphism of $\pi_{1}\left(S_{g}\right)$ is induced by a homeomorphism of $S_{\mathrm{g}}$. If $h$ is a homeomorphism $h: S_{\mathrm{g}} \rightarrow S_{\mathrm{g}}$ such that $h_{*}=M$ and $\lambda: S^{1} \rightarrow S_{\mathrm{g}}$ is a simple closed curve representing $\alpha_{1}$ then $h \lambda$ is a simple closed curve representing $X$. 
Conversely, suppose $\lambda \in \pi_{1}\left(S_{\mathrm{g}}\right)$ contains a simple closed curve and $\lambda$ represents $X$ in $H_{1}\left(S_{\mathrm{g}}\right)$. Since $\lambda$ represents $X \neq 0, \lambda$ contains a non separating simple closed curve. From results of Zieschang [3], there exists an automorphism $\alpha: \pi_{1}\left(S_{\mathrm{g}}\right) \rightarrow \pi_{1}\left(S_{\mathrm{g}}\right)$ mapping $a_{1}$ to $\lambda$. Then $\alpha_{*}$ is a symplectic automorphism and maps $\alpha_{1}$ to $X$. Therefore $X$ is a part of a symplectic basis, hence a basis, so the $\operatorname{gcd}\left\{p_{1}, \ldots, q_{\mathrm{g}}\right\}$ must be one.

Let $w=w\left(a_{1}, \ldots, b_{g}\right) \in \pi_{1}\left(S_{g}\right)$ and let $N\left(w, a_{1}\right)=$ sum of the exponents (positive and negative) of occurrences of $a_{i}$ in $w$. Similarly define $N\left(w, b_{i}\right)$.

Corollary. If $\operatorname{gcd}\left\{N\left(w, a_{1}\right), N\left(w, a_{2}\right), \ldots, N\left(w, b_{g}\right)\right\} \neq 1$ then the coset $w\left[\pi_{\mathrm{g}}, \pi_{\mathrm{g}}\right]$ does not contain a simple closed curve.

Proof. The image of $w$ in $H_{1}\left(S_{g}\right)$ is $\sum_{1}^{q}\left[N\left(w, a_{i}\right) \alpha_{i}+N\left(w, b_{i}\right) \beta_{i}\right]$ and the coset $w\left[\pi_{\mathrm{g}}, \pi_{\mathrm{g}}\right]$ contains all possible representatives of the image of $w$.

\section{REFERENCES}

1. W. Magnus, A. Karrass, and D. Solitar, Combinatorial Group Theory, Interscience, New York, London, 1966.

2. J. Nielsen, Untersuchungen zur Topologie des gescholossenen Zweiseitigen Flachen I, Acta Math. 50 (1927), pp. 184-358.

3. H. Zieschang, Algorithmen für einfache Kurven auf Flächen: Math. Scand., 17 (1965), 17-40.

UNIVERSITY OF MARYLAND

College Park, Maryland 